PREVENTION

\title{
Reducing the risk of sexually transmitted infections in genitourinary medicine clinic patients: a systematic review and meta-analysis of behavioural interventions
}

\author{
D J Ward, B Rowe, H Pattison, R S Taylor, K W Radcliffe
}

Sex Transm Infect 2005;81:386-393. doi: 10.1136/sti.2004.013714

See end of article for authors' affiliations

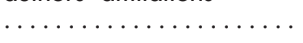

Correspondence to: Derek J Ward,

Birmingham and Solihull Health Protection Unit, Bartholomew House, 142 Hagley Road, Edgbaston, Birmingham B16 9PA, UK; derek.ward@hobtpct.nhs. uk

Accepted for publication 4 February 2005
Objectives: Are behavioural interventions effective in reducing the rate of sexually transmitted infections (STls) among genitourinary medicine (GUM) clinic patients?

Design: Systematic review and meta-analysis of published articles.

Data sources: Medline, CINAHL, Embase, PsychINFO, Applied Social Sciences Index and Abstracts, Cochrane Library Controlled Clinical Trials Register, National Research Register (1966 to January 2004). Review methods: Randomised controlled trials of behavioural interventions in sexual health clinic patients were included if they reported change to STI rates or self reported sexual behaviour. Trial quality was assessed using the Jadad score and results pooled using random effects meta-analyses where outcomes were consistent across studies.

Results: 14 trials were included; 12 based in the United States. Experimental interventions were heterogeneous and most control interventions were more structured than typical UK care. Eight trials reported data on laboratory confirmed infections, of which four observed a greater reduction in their intervention groups (in two cases this result was statistically significant, $p<0.05$ ). Seven trials reported consistent condom use, of which six observed a greater increase among their intervention subjects. Results for other measures of sexual behaviour were inconsistent. Success in reducing STIs was related to trial quality, use of social cognition models, and formative research in the target population. However, effectiveness was not related to intervention format or length.

Conclusions: While results were heterogeneous, several trials observed reductions in STI rates. The most effective interventions were developed through extensive formative research. These findings should encourage further research in the United Kingdom where new approaches to preventing STIs are urgently required.
$\mathrm{R}$ ecent reports highlight dramatic increases in sexually transmitted infections (STIs) in the United Kingdom ${ }^{1}$ alongside substantial increases in sexual risk behaviour. ${ }^{3}$ The government has responded with a national strategy for sexual health and HIV, which aims to improve the evidence base for prevention and sets targets for reducing new infections. ${ }^{4}$ Increased infection rates have placed considerable pressure on genitourinary medicine (GUM) clinics, ${ }^{36}$ which recorded over 1.5 million clinical episodes in England and Wales during 2002. ${ }^{1}$ Re-infection and re-attendance are thought to be common, but are not identified in routine data. A survey in England estimated that $44 \%$ of those diagnosed with gonorrhoea during 1996 had previously attended a GUM clinic. ${ }^{7}$ Reducing rates of re-infection among clinic patients by promoting behaviour change could contribute to current policy goals and improve public health.

We aimed to systematically review the evidence that behavioural interventions could reduce STIs in GUM clinic patients. A recent publication by the Health Development Agency $^{8}$ identified two high quality systematic reviews that considered randomised controlled trials (RCTs) of behavioural interventions in clinical settings that reported changes to STI rates. ${ }^{910}$ Both observed considerable heterogeneity between trials and concluded that the effectiveness of interventions was related to their development through formative research. We sought to build upon this previous work using a systematic approach to identify trials, an explicit approach to appraising trial validity, and quantitative methods to explore possible sources of heterogeneity. ${ }^{11}{ }^{12} \mathrm{We}$ also sought to broaden the scope of existing reviews by considering measures of sexual behaviour change, relating these to infection risk.

\section{METHODS \\ Identification of trials}

We searched seven databases of completed and ongoing research during January 2004 with no date or language restrictions (Medline, CINAHL, Embase, PsychINFO, Applied Social Sciences Index and Abstracts, Cochrane Library Controlled Clinical Trials Register, and the National Research Register). Searches used terms describing sexually transmitted infections or sexual behaviour combined (AND) with terms for prevention, health promotion, behavioural interventions, counselling, and psychological therapies. A full version of this report is available online. ${ }^{12}$

\section{Inclusion and exclusion criteria}

We included RCTs of behavioural interventions that aimed to reduce the risk of STIs in patients attending GUM or equivalent clinics. ${ }^{12}$ Infection rates were the primary outcome of interest, but we also included trials describing changes to self reported sexual behaviour. We excluded trials that provided educational materials alone, or reported only knowledge, attitudes, or behavioural intentions. Two reviewers independently assessed trials for inclusion using a standardised form. ${ }^{12}$

Abbreviations: GUM, genitourinary medicine; ITT, intention to treat; $M D$, mean difference; RCTs, randomised controlled trials; RR, relative risk 


\section{Trial quality}

Two reviewers independently assessed trial quality using a standardised form based on the Jadad scoring system. ${ }^{12} 13$ This considers the adequacy of randomisation, concealment, masking, completeness of follow up, and the use of intention to treat (ITT) analyses.

\section{Data abstraction and analysis}

All data were abstracted using a standardised form. ${ }^{12}$ STI diagnoses were ascertained from laboratory or clinic records, or the results of screening visits. Sexual behaviour change was ascertained from self completed questionnaires or interviews. Dichotomous outcomes were expressed as relative risks (RR) while results for continuous outcomes were expressed as the differences between intervention and control groups' mean within-subject change from baseline (mean difference, MD). ${ }^{12}{ }^{14}$ For infection related outcomes and sexual risk behaviours, a RR less than 1 or a negative MD indicates a lower rate of infections or risk taking in the intervention group relative to control. In contrast, for protective behaviours a RR greater than 1 or a positive MD indicates greater adoption of the protective behaviour in the intervention group relative to control. Infection related outcomes were analysed as ITT while behavioural outcomes represent on-treatment analyses (in many cases no information was reported on those lost to follow up).

Where outcome data were consistently available across studies, results were pooled using random effects metaanalyses (Review Manager, version 4.2, Cochrane Collaboration, 2003). Heterogeneity was explored with stratified meta-analyses using covariates defined a priori (study population, intervention characteristics, and trial quality). ${ }^{12}$

\section{RESULTS}

\section{Search for trials}

We included 31 papers reporting 14 trials (identified by principal citation, fig 1). ${ }^{12}$ Most rejected RCTs did not recruit patients from a relevant setting. Initial agreement on inclusion was good $(\kappa=0.70)$ with disagreements resolved by discussion.

\section{Study characteristics \\ Population and setting}

Most trials were conducted in the United States (table 1). Two trials recruited over $60 \%$ of all participants. ${ }^{15}{ }^{16}$ Four trials recruited only males ${ }^{17-20}$ and three only females. ${ }^{21-23}$ Three trials focused on adolescents or young women. ${ }^{212326}$ Most trials restricted entry to those with a recently diagnosed STI, ${ }_{17}^{18} 20-2527$ and/or reporting high risk sexual behaviours. ${ }^{16} 1826$ The largest trial did not restrict entry on these grounds and reported a $32 \%$ baseline STI rate. ${ }^{15}$

\section{Trial quality}

Jadad scores were low ( $\leqslant 3$ out of 5 ) reflecting an expected lack of masking and limited descriptions of trial methods. ${ }^{17} 2126-28$ Recruitment, adherence, and follow up rates were low (weighted mean $45 \% \mathrm{n}=12,66 \% \mathrm{n}=10$, and $69 \%$ $\mathrm{n}=12$ ), especially for interventions lasting over two sessions. Recruitment was greatest for small group interventions. Five trials reported differential follow up rates, ${ }^{17} 18232426$ though

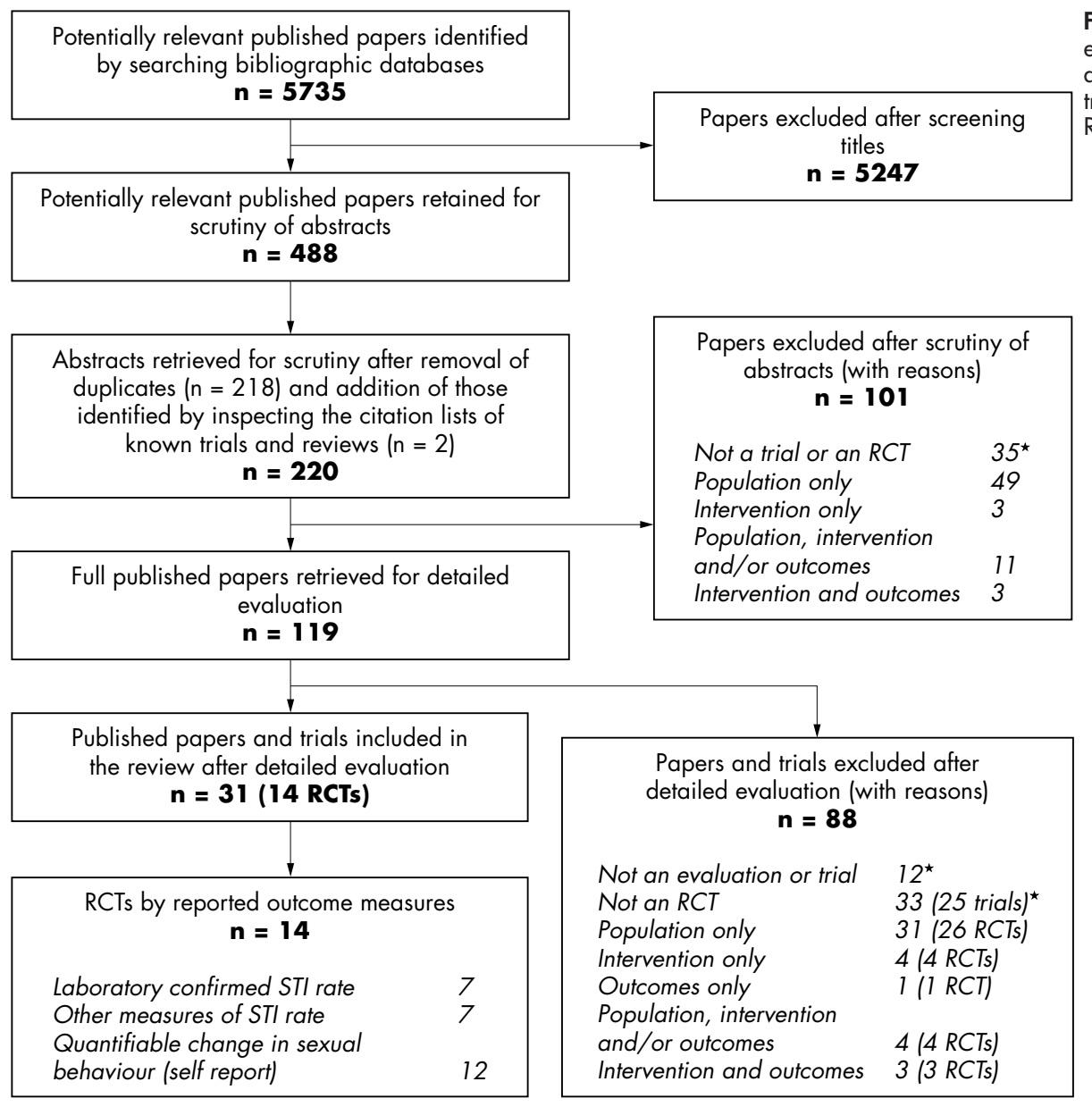

Figure 1 Flow of trials included and excluded from this review. ( $*$ No further details reported on those papers or trials not reporting evaluations or RCTs.) 

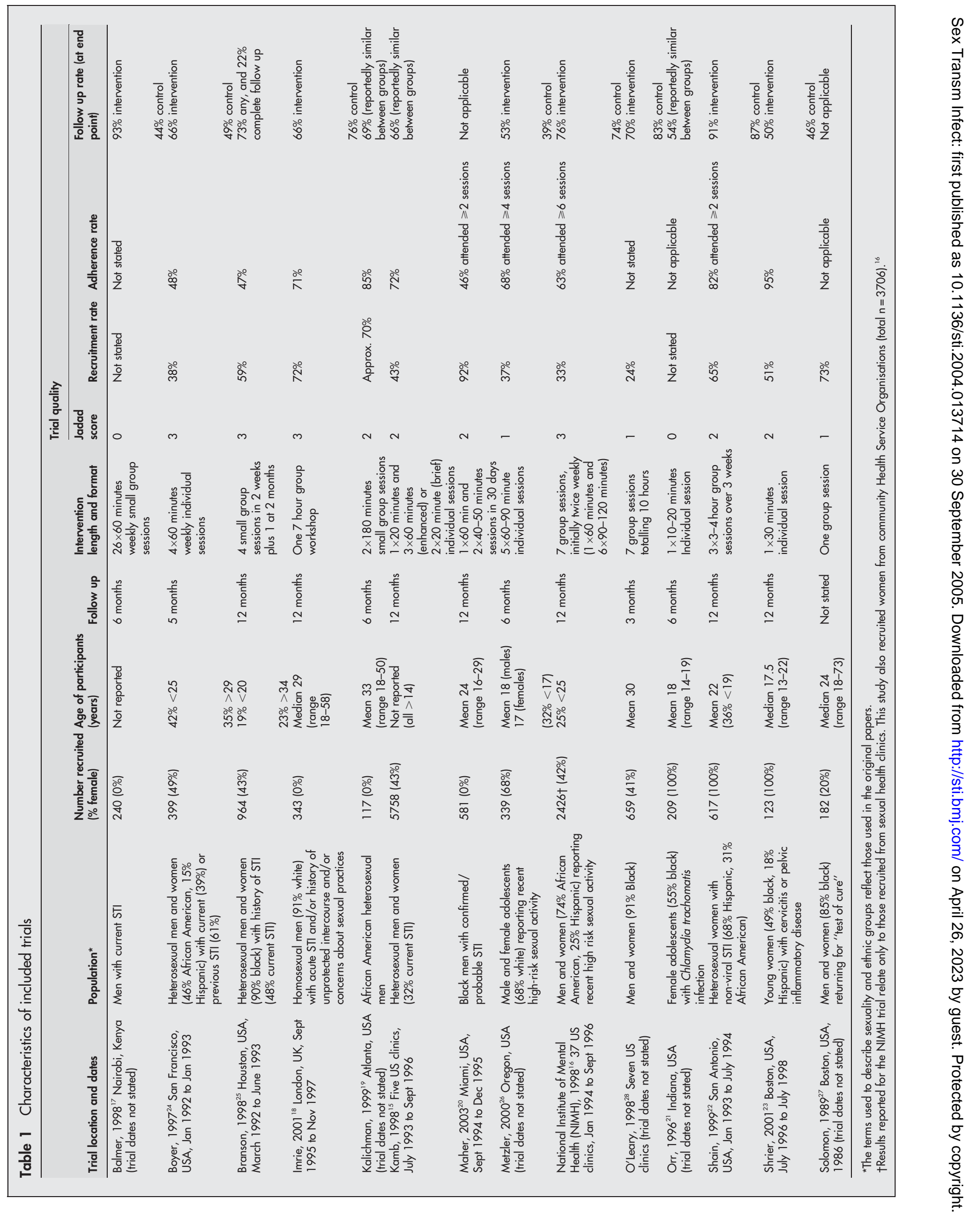


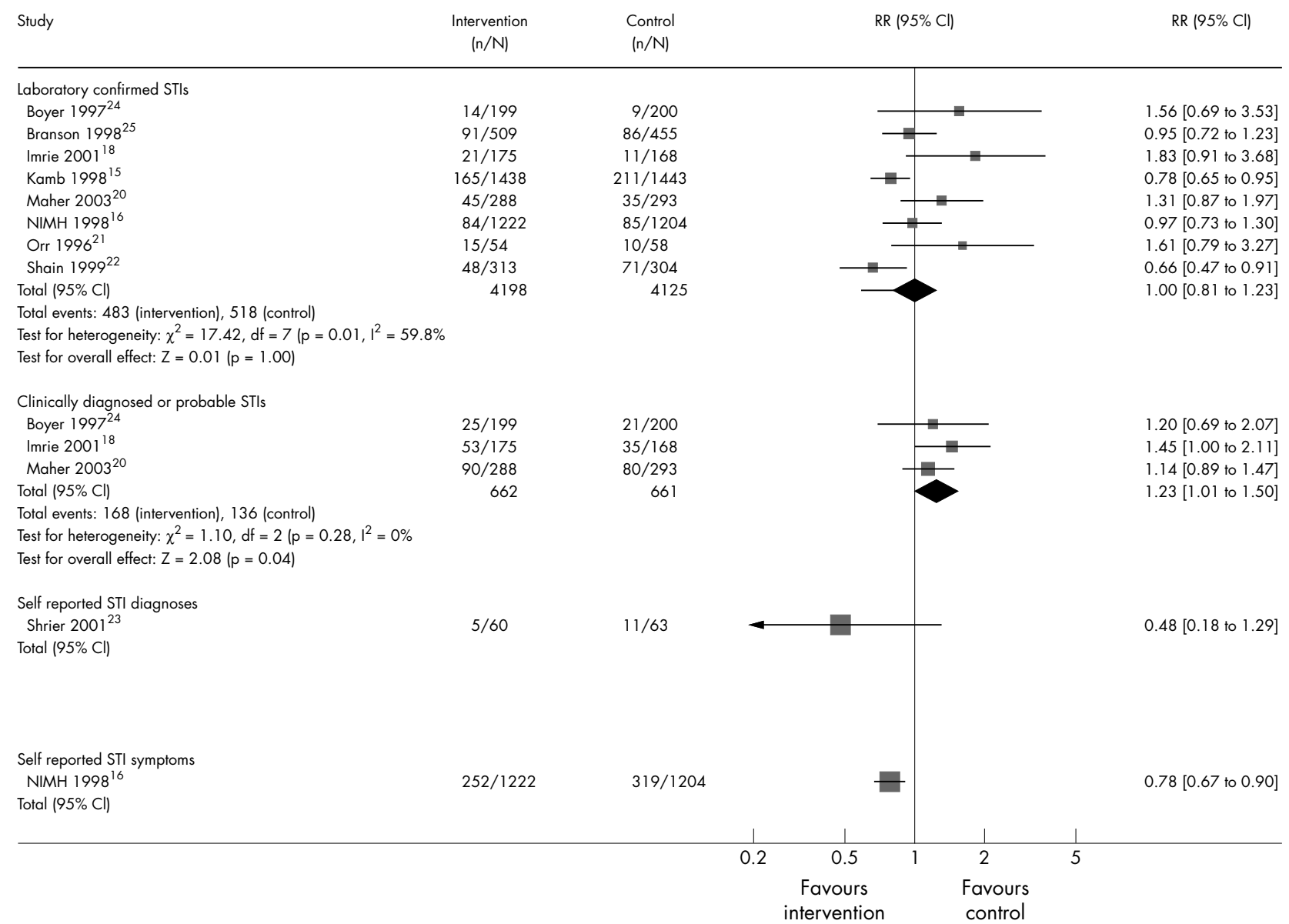

Figure 2 Results for laboratory confirmed STIs, clinically diagnosed STIs, self reported STIs, and self reported symptoms at trial end points. Cl, confidence interval; $n$, number of events; $N$, number of participants; RR, relative risk. Laboratory confirmed STI includes gonorrhoea, chlamydia, syphilis, HIV, trichomonas (two trials ${ }^{1422}$ ), non-specific urethritis (one trial ${ }^{14}$ ), chancroid and lymphogranuloma venereum (one trial ${ }^{18}$ ). Clinically diagnosed STIs also includes non-specific urethritis ${ }^{16} 1822{ }_{23}$ or mucopurulent cervicitis, ${ }^{22}$ pelvic inflammatory disease, ${ }^{22}$ trichomonas, first $^{23}$ presentations of genital warts, herpes simplex, or hepatitis B infection, ${ }^{16}{ }^{23}$ scabies, or pediculosis pubis, ${ }^{23}$ and presumptive treatment of either gonorrhoea or chlamydia on clinical grounds. ${ }^{18} 22$ Results for the trial by Kamb relate to the enhanced intervention. ${ }^{13}$ Equivalent results for the brief intervention are RR 0.82 (95\% Cl 0.68 to 0.99 ).

the group with greatest attrition was inconsistent. We labelled five trials as higher quality using a post hoc definition that required a Jadad score of two or three, masking of outcome assessors (seven trials), and nondifferential follow up..$^{15192022}$

\section{Experimental and control interventions}

Intervention format and length varied considerably (see table A on STI website www.stijournal.com/supplemental). One trial also compared an enhanced intervention with a brief one based on US best practice guidelines for individualised risk reduction counselling. ${ }^{152} 30$ Most other US trials adapted these guidelines for their control interventions, which are more structured and detailed than usual care currently provided in UK GUM clinics. ${ }^{12}$

Experimental interventions explored many similar themes, including risk perception, barriers to safer sex, and triggers to unsafe sex. Individually based interventions concentrated on accepting and negotiating condom use $\mathrm{e}^{21} 23$ and personal goal setting, ${ }^{15} 202426$ using roleplay to develop skills. ${ }^{24} 26$ In addition, group interventions explored self esteem in the context of social expectations ${ }^{16} 1825$ and increasing self efficacy..$^{16228}$ Modelling behaviours and skills was an important aspect of many interventions. ${ }^{16} 1719222528$

The two largest trials cited the theory of reasoned action and, less specifically, social cognition theory as the basis for their interventions. ${ }^{15} 16$ Social cognition theory asserts that attitudes to a behaviour, perceived support for that behaviour, and self efficacy determine intentions and subsequent actions. ${ }^{31}$ Other investigators also cited cognitive models such as the health belief model ${ }^{21}$ and AIDS risk reduction model, ${ }^{22}{ }^{24}$ which encourage participants to label themselves as vulnerable, commit to change, take action, and maintain change. ${ }^{32}$ Three trials cited the information motivation behavioural skills model, ${ }^{192526}$ which asserts that personalised information, motivation, and behavioural skills are prerequisites of adopting protective behaviours. ${ }^{31}$ Finally, two trials cited the transtheoretical model of behaviour change. ${ }^{1822}$ This represents change in terms of ordered stages allowing health promotion messages to be tailored to an individual's readiness to change.

\section{Trial results and quantitative data synthesis Laboratory confirmed STIs}

Four out of nine trials considering this outcome observed a greater reduction in their intervention groups relative to controls, ${ }^{15} 16^{2225}$ a result that was statistically significant $(\mathrm{p}<0.05)$ for two. ${ }^{15}{ }^{22}$ Pooled results do not indicate an overall effect (RR 1.00 (95\% confidence interval 0.81 to 1.23$)$ ). However, visual examination of the forest plot and statistical testing suggests heterogeneity between trials that prevents over-reliance on summary measures of effect (fig 2, 


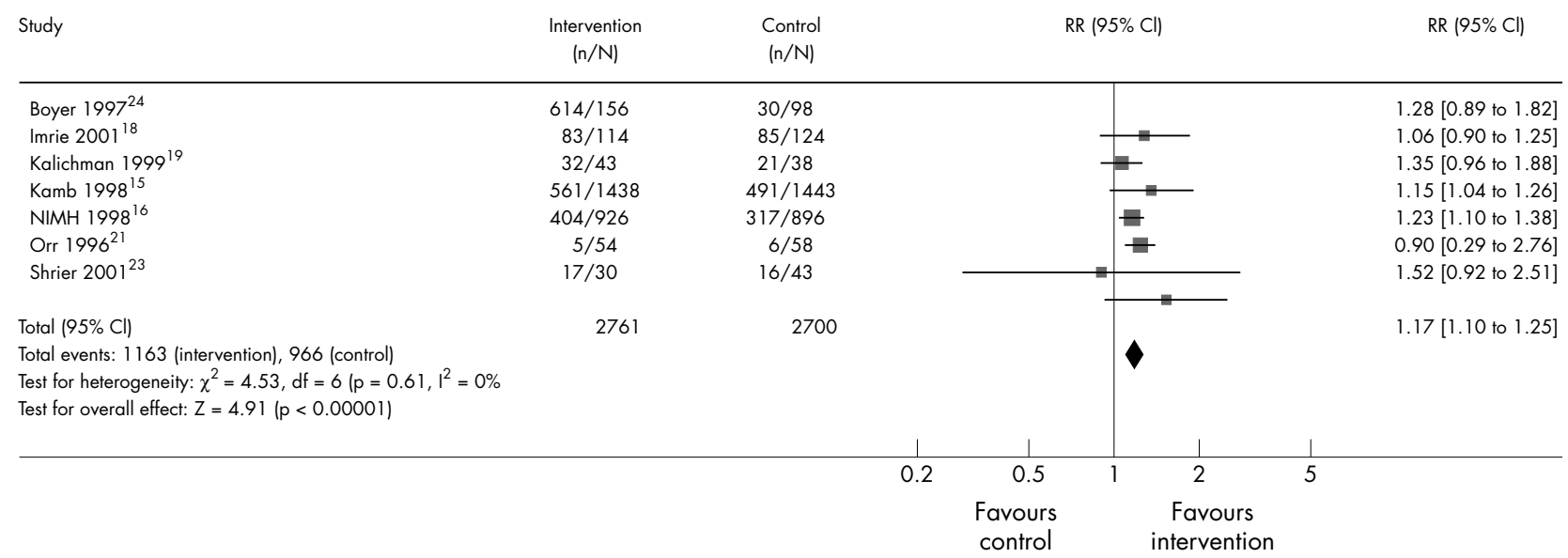

Figure 3 Results for consistent condom use (condoms used (nearly) always or no unprotected sex) at trial end points. Cl, confidence interval; $\mathrm{n}$, number of events; $N$, number of participants; RR, relative risk. Outcomes refer to self reported sexual behaviour for the previous $1-12$ months. Results for Imrie relate to the previous 1 month. ${ }^{16}$ Equivalent results for 12 months were RR 1.23 (95\% Cl 0.93 to 1.62 ).

$\mathrm{p}=0.001)$. In addition, a funnel plot of these results suggests evidence of publication bias (see fig A on STI website www.stijournal.com/supplemental, $\mathrm{p}=0.003$ Egger test). One further trial also reported a statistically significant effect on reducing gonorrhoea and urethral discharge, but did not present the original data (reported RRs 0.39 and 0.36 ). ${ }^{17}$

Trials reporting significant effects had among the greatest adherence and follow up rates. ${ }^{1522}$ Generally, high quality trials were more likely to report a protective effect, though pooled results were not significantly different (high quality RR 0.89 (0.70 to 1.12), low quality RR 1.29 (0.89 to 1.87))..$^{15} 162022$ Pooled results for trials enrolling both sexes $^{15} 162425$ (RR $0.90(0.78$ to 1.04$)$ ) or young women ${ }^{21} 22$ (RR 0.97 (0.41 to 2.32)) were greater than results for the remaining trials. ${ }^{18} 20$

Intervention format or length was not associated with trial results (group based RR 0.94 (0.70 to 1.25) versus individually based RR 1.16 (0.76 to 1.75$))$. Similarly, Kamb found that both brief and enhanced interventions were equally effective when compared to a short information session. ${ }^{15}$ We did find evidence that the theoretical basis of interventions was related to effectiveness, though differences in pooled results were not statistically significant (social cognition models RR 0.91 (0.71 to 1.16 ) versus other trials RR 1.19 $(0.84$ to 1.68$))$. In addition, extensive formative research, including interviews, focus groups, input from community representatives, and pilot testing, was reported by the four most effective trials..$^{15} 162225$

\section{Clinically diagnosed STIs}

Four studies considered clinically diagnosed STIs (fig 2). One trial found no reduction in any individual diagnosis ${ }^{25}$ while others observed greater STI rates in their intervention groups compared to controls. ${ }^{18} 2024$ Pooling of these results suggests an overall effect (RR 1.23 (1.01 to 1.50)) and there was no evidence of statistical heterogeneity $(\mathrm{p}=0.58)$.

\section{Self reported STIs}

Two trials considered self reported diagnoses and found no significant effects. ${ }^{23}{ }^{26}$ However, one trial observed an effect on self reported symptoms that was greater than that for laboratory confirmed infections. ${ }^{16}$

\section{Self reported condom use}

Six out of seven trials reporting consistent condom use (condoms always used or used at every sexual encounter) observed a greater increase in their intervention groups than controls. ${ }^{151618192324}$ Examination of the forest plot and statistical testing do not indicate heterogeneity between trials (fig 3, p =0.61) and the pooled result suggests an overall effect (RR $1.17(1.10$ to 1.25$))$. In contrast with laboratory confirmed infections, there is no evidence of publication bias (see fig B on STI website www.stijournal. com/supplemental, $\mathrm{p}=0.98$ Egger test).

Pooled results do not suggest that effects were related to trial quality (high quality RR 1.19 ( 1.11 to 1.28$) v$ low quality RR 1.12 (0.97 to 1.29)), intervention format (group based RR 1.18 (1.05 to 1.33 ) $v$ individually based RR 1.16 (1.06 to 1.28)), intervention length, the use of theory, or formative research. Results for trials recruiting females (RR $1.40(0.88$ to 2.20)) were greater than for trials recruiting mixed populations (RR 1.19 ( 1.10 to 1.27$)$ ) or males alone (RR $1.14(0.92$ to 1.42$))$.

Five trials considered the proportion of sexual encounters protected by condoms. ${ }^{16} 19222428$ Two found increased rates in intervention subjects relative to controls (table 2). Other trials reported inconsistent effects on refusing unsafe $\operatorname{sex}^{1928}$ and using condoms with recent partners..$^{23}$

\section{Sexual partners}

Five out of seven trials reported fewer sexual partners among their intervention groups relative to controls (table 2), ${ }^{17} 22242628$ while two reported fewer partners among controls. ${ }^{1925}$ Two of these trials also reported a reduction in sexual encounters with strangers or non-monogamous partners, ${ }^{26}$ and more avoidance of sex with partners suffering from known STIs. ${ }^{22}$ However, another intervention had no effect on homosexual men reporting unprotected sex with a partner of different or unknown HIV status. ${ }^{18}$

\section{DISCUSSION}

Several trials of behavioural interventions in sexual health clinic patients observed a greater reduction in laboratory confirmed STIs among their intervention groups relative to controls despite using different intervention formats in different populations. Successful trials were larger and of higher quality than those reporting no reduction in infection rates, and the most effective interventions were based on social cognition or related theoretical models and developed through extensive periods of formative research. We also found evidence that behavioural interventions increase consistent condom use, though effects on other aspects of sexual behaviour were inconsistent. There was no evidence 


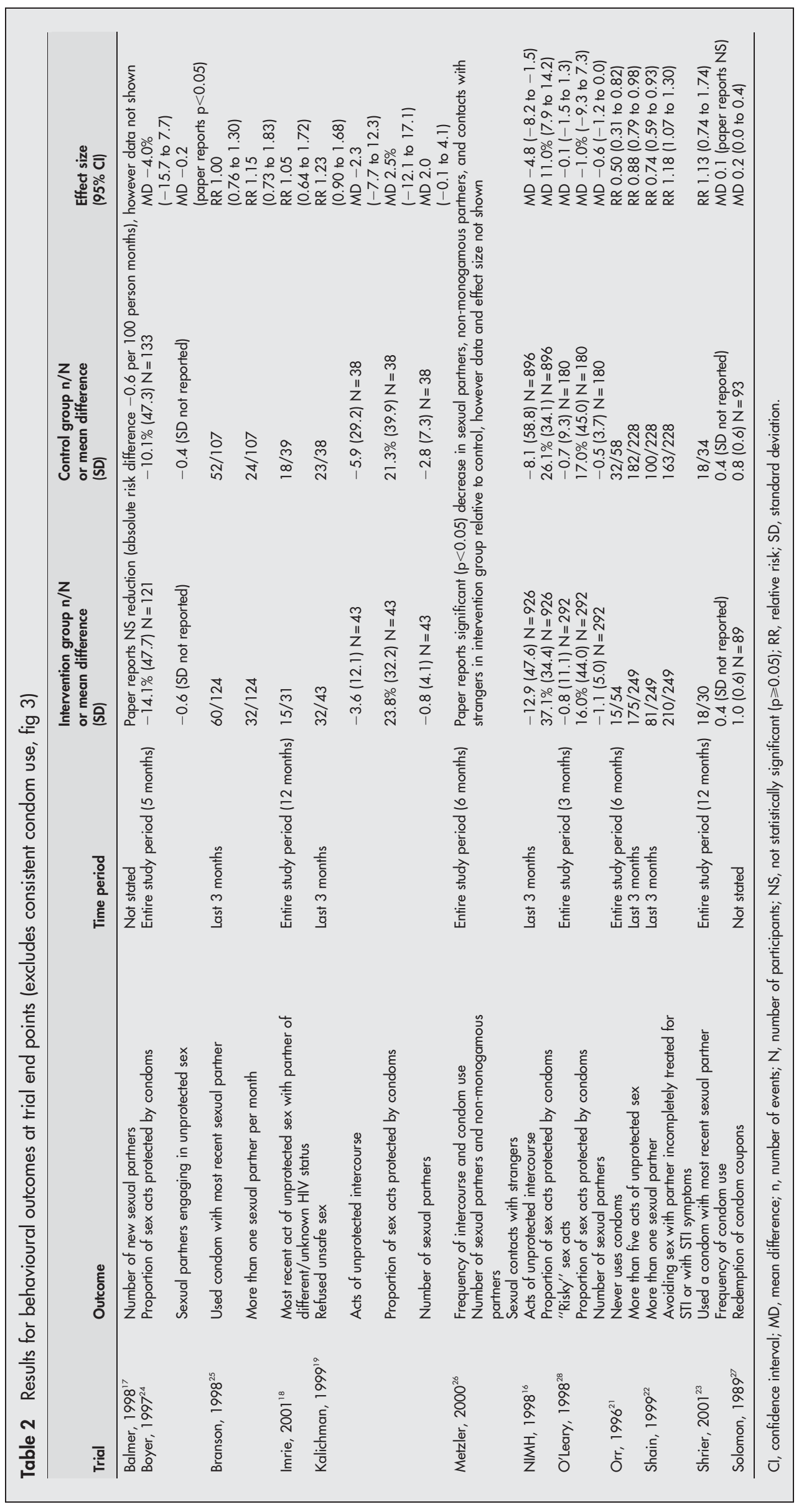




\section{Key messages}

- Interventions that could modify behaviour in GUM clinic patients (a high risk group) could play an important part in reducing recurrent STIs and thereby improve public health

- Two large high quality trials of behavioural interventions have demonstrated statistically significant reductions in STI rates despite using different intervention formats and targeting different populations

- Most trials also demonstrate an increase in self reported condom use, though effects on other aspects of sexual behaviour are inconsistent

- The most successful interventions were based on social cognition models and underwent careful development through extensive formative research. These results should be used to direct future research in a UK setting

that an intervention's success in reducing infections or increasing consistent condom use was related to its format or length.

One trial observed no difference in effect between a multiple session enhanced intervention and a two session brief one. ${ }^{15}$ This brief intervention was similar to the control intervention used by many trials, ${ }^{20-22} 2425$ including one that successfully reduced infection rates. ${ }^{22}$ In effect, the control groups in these trials received an intervention that goes beyond current UK practice, making it difficult to generalise results or transfer effect estimates to a UK settings.

The quality of identified studies was frequently poor. Masking is difficult to achieve in trials of behavioural interventions, but we did consider masking of outcome assessors in our quality assessment as this may reduce bias. ${ }^{33}$ We included only RCTs in this review, which could have resulted in the omission of potentially relevant information. However, a suitable unbiased control group is essential to avoid ascribing sexual behaviour change to the effects of the intervention rather than the diagnosis of an STI itself. ${ }^{32}{ }^{33} \mathrm{In}$ addition, we found evidence of publication bias for the primary outcome (laboratory confirmed infections), though we attempted to identify all publicly funded research in the United Kingdom.

Two previous reviews also reported inconsistent results, though they suggest that adherence rates and the development of an intervention through formative research were predictors of success. ${ }^{9}{ }^{10}$ We identified five trials not included in these reviews, ${ }^{17} 20232628$ four of which report infection related outcomes, yet our findings suggest similar factors are important to an intervention's effectiveness. Formative research seeks to identify the behaviours, motivations, and beliefs within the target population that lead to increased risk, and link these to the key elements of an intervention. ${ }^{10} 32$ The format, setting, delivery, and acceptability of the intervention can then be explored through pilot testing. ${ }^{32}$ Theory may provide a framework for this process, though models do not specify the inclusion of particular elements within an intervention, and we also found evidence that effective interventions were more likely to be based on theoretical models relating behaviour to individual cognitions.

Trials appeared more effective at increasing (consistent) condom use than reducing infections. This could represent a chance event, or bias resulting from the different methods of ascertainment. Alternatively, small changes in condom use may be an insensitive marker of overall STI risk, which is the sum of many behavioural factors. ${ }^{10}{ }^{32}$ At the population level, increased condom use has been accompanied by increases in other risky sexual activities and a marked rise in infections. ${ }^{3}$ Further analysis of the NIMH trial data also suggests that the number of sexual partners remains an important risk factor for gonorrhoea infection regardless of changes to condom use. $^{34}$ However, too few trials reported both behavioural outcomes alongside infection rates to explore this issue further.

This review highlights a need for research on behavioural interventions in a UK setting, where new approaches to reducing infection rates are urgently required. Heterogeneity between trials for the primary outcome of interest means that we cannot rely on simple summary measures of effect, but there is evidence that interventions can successfully change sexual behaviour and reduce infection rates if appropriately developed and targeted to a population. The success of interventions appears unrelated to their intensity. This is important, as the number of sessions is likely to be a major factor in determining cost. However, even the briefest intervention shown to be effective could not be introduced into routine GUM practice without further investment in premises, staff, and training. Information on the likely effect size, acceptability, and cost effectiveness of introducing behavioural interventions into the United Kingdom will be critical to those developing new services. However, this information cannot be simply extrapolated from existing evidence, which mainly derives from the United States. We propose that future studies should develop their experimental interventions by tailoring the approaches shown to be most effective in US settings, and crucially inform this process by a period of formative research that incorporates qualitative approaches. While this is likely to be time consuming and costly, without such an approach any proposed intervention is unlikely to be successful.

\section{CONTRIBUTORS}

The idea for the review was proposed by KWR, and the review was planned by DJW and RST with assistance from all authors; DJW developed the search strategy and together with BR undertook the searches, appraised the articles, and extracted the data; DJW analysed the data with the help of RST and HP; writing up the report was principally done by DJW with input from all members of the review team; in particular, RST advised on systematic review methods; HP advised on behavioural interventions, their theoretical basis and application, and KWR advised on sexual health and GUM clinic practice.

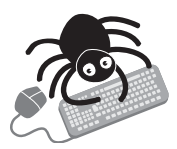

One supplementary table and two figures are on the STI website (www.stijournal.com/supplemental)

\section{Authors' affiliations}

D J Ward, B Rowe, Public Health, South Worcestershire Primary Care Trust, Worcester, UK

H Pattison, Department of Psychology, Aston University, Birmingham, UK

R S Taylor, Public Health and Epidemiology, The University of Birmingham, Birmingham, UK

K W Raddliffe, Genitourinary Medicine, Heart of Birmingham Teaching Primary Care Trust, Birmingham, UK

Funding: All contributors undertook this review as part of their normal employment and did not receive specific funding for this study, which was carried out independently of their employers.

Competing interest: The authors have no competing interests.

Ethical approval: Ethical approval was not required for this study. DJW and BR are employed on the West Midlands Public Health Higher Specialist Training Scheme. At the time of undertaking this study, both were working at South Worcestershire Primary Care Trust. HP is 
employed by the School of Life and Health Sciences at Aston University, while RST is employed by the University of Birmingham and funded by the West Midlands Regional Public Health levy. KWR is employed by the Heart of Birmingham Teaching Primary Care Trust and works as a consultant in genitourinary medicine at the Whittall Street Clinic.

\section{REFERENCES}

1 Health Protection Agency, SCIEH, ISD, National Public Health Service for Wales CDSC Northern Ireland, and the UASSG. Renewing the focus. HIV and other sexually transmitted infections in the United Kingdom in 2002. London: Health Protection Agency, 2003.

2 PHLS, DHSS\&PS, and the Scottish ISD(D)5 Collaborative Group. Sexually transmitted infections in the UK: new episodes seen at genitourinary medicine clinics, 1991 to 2001. London: Public Health Laboratory Service, 2002.

3 Johnson AM, Mercer CH, Erens B, et al. Sexual behaviour in Britain: partnerships, practices, and HIV risk behaviours. Lancet 2001 358:1835-42.

4 Department of Health. The national strategy for sexual health and HIV. London: Department of Health, 2001.

5 Department of Health. The national strategy for sexual health and HIVimplementation action plan. London: Department of Health, 2002.

6 House of Commons Health Committee. Sexual Health. Volume I: report, together with formal minutes. Third report of session 2002-03 (HC69-1). London: House of Commons, 2003.

7 Hughes G, Andrews N, Catchpole M, et al. Investigation of the increased incidence of gonorrhoea diagnosed in genitourinary medicine clinics in England, 1994-6. Sex Transm Infect 2000;76:18-24.

8 Ellis S, Grey A. Prevention of sexually transmitted infections (STIs): a review of reviews into the effectiveness of non-clinical interventions. Evidence briefing. 1 st ed. London: Health Development Agency, 2004

9 Elwy AR, Hart GJ, Hawkes S, et al. Effectiveness of interventions to prevent sexually transmitted infections and human immunodeficiency virus in heterosexual men. Arch Intern Med 2002;162:1818-30

10 Stephenson JM, Imrie J, Sutton SR. Rigorous trials of sexual behaviour interventions in STD/HIV prevention: what can we learn from them? AIDS 2000; 14:s 115-24.

11 NHS Centre for Reviews and Dissemination, University of York. Undertaking systematic reviews of research on effectiveness. CRD Report Number 4. 2nd ed. York: NHS Centre for Reviews and Dissemination, University of York, 2001.

12 Ward DJ, Rowe B, Pattison $\mathrm{H}$, et al. Behavioural interventions to reduce the risk of sexually transmitted infections in genitourinary medicine clinic patients: a systematic review (www.pcpoh.bham.ac.uk/publichealth/wmhtac) reports.htm) Birmingham: West Midlands Health Technology Assessment Collaboration, University of Birmingham, 2004

13 Jadad AR, Moore RA, Carroll D, et al. Assessing the quality of reports of randomized clinical trials: is blinding necessary? Control Clin Trials 1996;17:1-12

14 Follmann D, Elliot P, Suh I, et al. Variance imputation for overviews of clinical trials with continuous response. J Clin Epidemiol 1992;45:769-73.

$15 \mathrm{Kamb}$ ML, Fishbein M, Douglas JMJ, et al. Efficacy of risk-reduction counseling to prevent human immunodeficiency virus and sexually transmitted diseases. A randomized controlled trial. JAMA 1998;280:1161-7.
16 National Institute of Mental Health (NIMH) Multisite HIV Prevention Trial Group. The NIMH Multisite HIV Prevention Trial: reducing HIV sexual risk behavior. Science 1998;280:1889-94.

17 Balmer DH, Gikundi E, Nasio J, et al. A clinical trial of group counselling for changing high-risk sexual behavior in men. Counselling Psychology Quarterly 1998; 11:33-43.

18 Imrie JM, Stephenson JM, Cowan FM, et al. A cognitive behavioural intervention to reduce sexually transmitted infections among gay men: randomised trial. BMJ 2001;322:1451-6.

19 Kalichman SC, Cherry C, Browne-Sperling F. Effectiveness of a video-based motivational skills-building HIV risk-reduction intervention for inner-city African American men. J Consult Clin Psychol 1999;67:959-66.

20 Maher JE, Peterman TA, Osewe PL, et al. Evaluation of a community-based organization's intervention to reduce the incidence of sexually transmitted diseases: a randomized controlled trial. Sex Transm Dis 2003;96:248-53.

21 Orr DP, Langefeld CD, Katz BP, et al. Behavioral intervention to increase condom use among high-risk female adolescents. J Pediatr 1996;128:288-95.

22 Shain RN, Piper JM, Newton ER, et al. A randomized, controlled trial of a behavioral intervention to prevent sexually transmitted disease among minority women. N Engl J Med 1999;340:93-100.

23 Shrier LA, Ancheta R, Goodman E, et al. Randomized controlled trial of a safer sex intervention for high-risk adolescent girls. Arch Pediatr Adolesc Med 2001; 155:73-9.

24 Boyer CB, Barrett DC, Peterman TA, et al. Sexually transmitted disease (STD) and HIV risk in heterosexual adults attending a public STD clinic: evaluation of a randomized controlled behavioral risk-reduction intervention trial. AIDS 1997; 1 1:359-67.

25 Branson BM, Peterman TA, Cannon RO, et al. Group counseling to prevent sexually transmitted disease and HIV: a randomized controlled trial. Sex Transm Dis 1998;25:553-60.

26 Metzler CW, Biglan A, Noell J, et al. A randomized controlled trial of a behavioral intervention to reduce high-risk sexual behavior among adolescents in STD clinics. Behavior Therapy 2000;31:27-54.

27 Solomon MZ, DeJong W. Preventing AIDS and other STDs through condom promotion: a patient education intervention. Am J Public Health 1989;79:453-8.

28 O'Leary A Ambrose TK, Raffaelli M, et al. Effects of an HIV risk reduction project in sexual risk behavior of low-income STD patients. AIDS Educ Prev 1998; 10:483-92.

29 Centers for Disease Control and Prevention. Technical guidance on HIV counseling. MMWR Recomm Rep 1993;42(RR-02)

30 Centers for Disease Control and Prevention. Sexually transmitted diseases treatment guidelines 2002. MMWR Recomm Rep 2002;51 (RR-06).

31 Fisher WA. A theory-based framework for intervention and evaluation in STD/HIV prevention. Canadian Journal of Human Sexuality 1997;6:105-11.

32 Bonnell C, Imrie J. Behavioural interventions to prevent HIV infection: rapid evolution, increasing rigour, moderate success. Br Med Bull 2001;58:155-70.

33 Stephenson J, Imrie J. Why do we need randomised controlled trials to assess behavioural interventions? BMJ 1998;316:611-13.

34 Pinkerton SD, Layde PMDW, Chesson HW, et al. All STDs are not created equal: an analysis of the differential effects of sexual behaviour changes on different STDs. Int J STD AIDS 2003;14:320-8. 